\title{
Avoiding Fumbles: Online Patient Handoff Training
}

Jack Wells, MD, MHA | Dena Higbee, EdS, MS, CHSE | Jen Doty, BSN | Elaine Louder, BS

PRiMER. 2020;4:32.

Published: 10/29/2020 | DOI: 10.22454/PRiMER.2020.984649

\section{Abstract}

Introduction: The COVID-19 pandemic required rapid curriculum adaptation to online delivery. Given the importance of accurate clinical patient handoffs, we adapted simulation-based medical student training in clinical patient handoffs. We scored the accuracy of the information students presented. We also elicited student feedback to determine their perceptions of the event and their evaluations of the effectiveness of the training.

Methods: Twenty-six third-year medical students participated online via Zoom. Students participated in groups of three or four students. They sequentially encountered a standardized patient. Clinical information and physical exam findings were handed off from student to student until the encounter was complete. The student group then debriefed with faculty. Students were evaluated based on the proportion of clinical information handed off to the following student. Students also evaluated the training session.

Results: The first student handoff included $73.4 \%$ of the available information. In subsequent handoffs, the percentage fell to $43 \%$. All students said they felt the training was helpful in practicing patient handoffs and interactions with colleagues. Negative student comments focused on perceived deficiencies in session planning and standardized patient training.

Conclusions: This rapidly developed simulation session demonstrated that clinical training in patient handoffs can be adapted to an online environment. In order to ensure success, faculty should carefully consider logistical adjustments required to transition from a patient contact setting to an online environment. Meticulous preparation and attention to detail will ensure that training is successful.

\section{Introduction}

The COVID-19 pandemic necessitated the rapid transition of clinical teaching to online delivery. Medical schools faced the challenge of adapting to noncontact teaching, and prioritizing curriculum adaptations. We prioritized medical student training in patient handoffs. The American Association of Medical Colleges (AAMC) has identified clinical case presentation as an entrustable professional activity. ${ }^{6}$ Since clinical handoff training in medical schools is often lacking, ${ }^{1-3}$ we adapted medical student training in clinical patient handoffs to an online setting. Medical errors can result from inaccurate handoff information, 3,4 
therefore medical students should receive training and experience in presenting clinical information. 5,6 Medical students at the University of Missouri-Columbia School of Medicine learn patient handoff skills in simulation sessions with standardized patients (SP). We adapted these to an online format. We scored the amount and accuracy of the information students presented. We also elicited student feedback to determine their perceptions of the event and their evaluations of the effectiveness of the training. We submit this description of a rapid adaptation of a live simulation session to an online setting.

\section{Methods}

Third-year medical students in the surgery clerkship participated in a patient handoff simulation. The simulations used the Zoom video communication platform. The SP presession training included usual appropriate history information. Additional format-specific training included describing physical findings verbally when asked.

Encounters consisted of a 30-minute online encounter with a SP complaining of abdominal pain, followed by a 15-minute debriefing. A faculty member monitored and scored all encounters and participated in the debriefing.

The students divided into groups of three or four. The first student obtained the history and presented to the second student. The second student performed the examination by verbalizing physical examination maneuvers, such as abdominal palpation or cardiopulmonary auscultation. They then presented the history and physical to the third student. The third student interpreted lab values, and consulted with the first two students to develop a differential diagnosis. The third student then presented the history, physical, and lab data to the fourth student who concluded the case with the patient and did not do a formal presentation. In a three-person group, the third student did not do a presentation, but did finish the session with the patient. During the encounters, students were in a waiting room until their turn. Following their encounter, all students returned to the waiting room. Finally, all students returned to the live session for a group debriefing with faculty.

The scoring tool is shown in Figure 1. Items on the checklist were marked if they were identified in the patient interview and simulated physical examination, and as they were relayed in the checkout. Each checkout was scored, and the results were recorded in Excel. The number of items transmitted in each checkout were scored as a percentage of the number of items available. The number of available items increased as the case encounter progressed with more information added. The number of items conveyed in checkout could vary between sessions depending on the number of students in the group. Students completed a survey evaluating the session. The University of Missouri Institutional Review Board designated this study exempt.

\section{Results}

Twenty-six medical students participated. All students participated in each step of the simulation. Overall, students presented less information as the session progressed. The results are shown in Table 1. Information available with each student encounter increased in each step with the addition of physical findings and laboratory information.

Following the event, students received a survey evaluating the session. The survey consisted of four questions rating the session scored on a Likert scale, followed by a section for comments about the session. The survey had a $50 \%$ response rate (Table 2 ). This may have been because the surveys were sent 
out 1 day after the event. The students who failed to respond were not resurveyed.

Most of the students responding said they felt that the event was helpful in practicing patient handoffs, and in interacting with colleagues. Two students felt that the sessions were not helpful (Figure 2).

Student comments regarding the usefulness of the event were mixed. The comments reflect a perception of inadequate SP preparation for the session. Students also felt that they were not well prepared and had poor understanding of expectations. Students also felt that they were given insufficient information about the cases prior to encountering the patient. Students also expressed uncertainty in eliciting physical findings. They also felt that the SPs appeared less prepared than for live encounters, and seemed uncertain in responding to some questions. A likely reason for these comments could be the relatively short preparation time for this session and the setting, which is different than the students and the SP routinely use.

\section{Conclusions}

Online SP encounters can provide medical students practice with clinical patient handoffs. Consistent with prior research, ${ }^{7}$ the fidelity of the information presented decreased with time and increasing number of handoffs. This decrease may be due to sequential addition of information, or uncertainty in the online setting.

Successful online adaptation of live teaching requires planning. This session was adapted to an online format rapidly. In planning such sessions, presession learner briefings should specify the process, procedures, and anticipated flow of the session. Appropriate procedures, such as asking the SP for responses, verbalized physical examination maneuvers, and requesting laboratory data, should be clear to the learners. SPs should also know how to respond appropriately. They should clearly understand how to provide historical information and to provide physical finding information.

Our session had several limitations. It was rapidly prepared with little on-site support, due to simulation center staff work restrictions. As a result, learners perceived that the SPs were not adequately prepared to provide information. Another limitation is the low number of student participants and low number of responses to the post session survey. The inability to perform physical examinations is a limitation and can significantly decrease the fidelity of the experience. This setting could be very effective in training in a scenario involving cognitive management of conditions and not requiring physical examination.

Clinical education in case presentations can occur online. Faculty should consider logistical adjustments required to transition from a patient contact setting to a virtual environment. Meticulous preparation and attention to detail will help ensure that training is successful.

\section{Tables and Figures}


Figure 1: Checklist Used in Scoring the Handoff Simulation

\begin{tabular}{|c|c|c|c|}
\hline \multicolumn{4}{|l|}{ Self-eval of what was stated (Learner's name): } \\
\hline $\begin{array}{llll}\text { Order of giving checkout: } & 1 & 2 & 3\end{array}$ & & & \\
\hline \multicolumn{4}{|l|}{ Checklist: Cholecystitis } \\
\hline History Taking & YES & NO & $\begin{array}{c}\text { Present } \\
\text { in } \\
\text { Checkout }\end{array}$ \\
\hline 1. Patient ID $(50$ ye $F)$ & 0 & 0 & \\
\hline 2. Onset of the abdominal pain ( 12 hours ago) & 0 & 0 & \\
\hline 3. Pain worse (nothing) & 0 & 0 & \\
\hline 4. Pain better (nothing-tried Mylanta with no relief) & 0 & 0 & \\
\hline 5. Quality of the pain (belly pain, discomfort - crampy) & $\mathrm{O}$ & 0 & \\
\hline $\begin{array}{l}\text { 6. Location of the pain (right upper abdomen started - now back below } \\
\text { right shoulder) }\end{array}$ & $\mathrm{O}$ & $\mathrm{O}$ & \\
\hline 7. Pain on a scale from 1-10 (currently at 7 ) & $\mathrm{O}$ & 0 & \\
\hline $\begin{array}{l}\text { 8. Related symptoms: Like what? fever }(y) \text {, chills (n), nausea }(y) \text {, } \\
\text { vomiting (no), loss of appetite }(y) \text {, constipation }(n) \text {, diarrhea }(n) \\
\text { bloating }(y) \text { belching }(y)\end{array}$ & $\mathrm{O}$ & 0 & \\
\hline 9. Timing of the pain (started after dinner of polish sausage) & $\mathrm{O}$ & $\mathrm{O}$ & \\
\hline 10. Lab values/Diagnostics & 0 & 0 & \\
\hline 11. Last meal (toast this am) & $\mathrm{O}$ & 0 & \\
\hline 12. Current medications (multi vitamin $-A L L-P C N$ ) & $\mathrm{O}$ & $\mathrm{O}$ & \\
\hline $\begin{array}{l}\text { 13. Previous episodes of similar pain (yes-not this bad-subsided over 2-3 } \\
\text { howrs) }\end{array}$ & $\mathrm{O}$ & $\mathrm{O}$ & \\
\hline 14. Past medical history (hysterectomy for fibroids 6 yrs ago) & $\mathrm{O}$ & $\mathrm{O}$ & \\
\hline $\begin{array}{l}\text { 15. Family medical history (father died } 64 \text { heart attack, mom alive } 72 \mathrm{hx} \text { - } \\
\text { breast cancer) }\end{array}$ & $\mathrm{O}$ & 0 & \\
\hline 16. Tobacco, drugs AND alcohol use (none) & 0 & 0 & \\
\hline $\begin{array}{l}\text { 17. Possible diagnosis.(Ulcer, GI, influenza, hemia, cholelithiasis vs } \\
\text { cholecystitis) }\end{array}$ & & & \\
\hline 18. Closed check out and explained next step & $\mathrm{O}$ & 0 & \\
\hline $\begin{array}{l}\text { - Complete the Yes/No column after relaying the patient information. } \\
\text { - Complete the "present in checkout" column after receiving the last chech }\end{array}$ & & & \\
\hline
\end{tabular}

Table 1: Percentage of Information Transmitted in Checkout $(\mathrm{N}=26)$

\begin{tabular}{|c|c|}
\hline Student Order* & Percent of Items Transmitted \\
\hline Student 1 & 73.4 \\
\hline Student 2 & 62.5 \\
\hline Student 3 & 48.3 \\
\hline
\end{tabular}

*The fourth student in the group ended the visit and did not do a checkout. 
Table 2: Student Feedback $(\mathrm{N}=13)$

\begin{tabular}{|l|c|c|}
\hline \multicolumn{1}{|c|}{ Response } & $n$ & $\%$ \\
\hline Strongly agree & 8 & 61.5 \\
\hline Agree & 5 & 38.5 \\
\hline Strongly disagree & 0 & 0 \\
\hline Disagree & 0 & 0 \\
\hline \multicolumn{1}{|c|}{ The Event Was Helpful in Learning and Practicing Professional Interactions With My Colleagues } \\
\hline Strongly agree & 7 & 53.8 \\
\hline Agree & 5 & 38.5 \\
\hline Strongly disagree & 1 & 14.3 \\
\hline Disagree & 0 & 0 \\
\hline \multicolumn{2}{|c|}{ The Event Was Helpful in Practicing Patient Interactions } \\
\hline Strongly agree & 7 & 53.8 \\
\hline Agree & 5 & 38.5 \\
\hline Strongly disagree & 1 & 8.3 \\
\hline Disagree & 0 & 0 \\
\hline \multicolumn{2}{|c|}{ I found the feedlback from the preceptors valuable } \\
\hline Strongly agree & 12 & 92.3 \\
\hline Agree & 1 & 7.7 \\
\hline Strongly disagree & 0 & 0 \\
\hline Disagree & 0 & 0 \\
\hline
\end{tabular}

Figure 2: Selected Student Comments From the Postsession Evaluation

"I just wish I had known more about what was going to happen beforehand. I had no idea what to expect or if any preparation was needed."

"A little more advance notice on what we were actually doing would have been nice. I did not know what the goal of the encounter was until the debrief."

"I think the physical exam portion needs to be improved/changed. I wish I had been given some form of document with vitals/PE findings. I found myself trying to ask the pt about physical exam findings and all she did was sit there and not respond. I think the standardized pt needs better training on what their role is/what info to provide us with during these zoom simulations."

"Just a little more information beforehand on what to expect."

"I think a few more details in the PE could be listed just so we have to convey more information. Also it may be helpful to let students who have already gone stay in the breakout room until the end so they can see what other members were tasked with (they stay muted)."

"I think it might be helpful to have team members continue to be in the room, but silent, so that they can also give feedback based on what they see. For instance, the person taking the Hx might be able to see how information they gave was either sufficient or insufficient to the task, or learn what things they might have missed to improve their work." 


\section{Corresponding Author}

Jack Wells, MD, MHA

M245 Medical Sciences Building, Department of Family and Community Medicine, School of Medicine, University of Missouri, Columbia, MO 65212. 573-999-6502. Fax: 573-642-3015.

wellsjack@health.missouri.edu

\section{Author Affiliations}

Jack Wells, MD, MHA - Department of Family and Community Medicine, School of Medicine, University of Missouri, Columbia, MO

Dena Higbee, EdS, MS, CHSE - Shelden Simulation Center, University of Missouri-Columbia

Jen Doty, BSN - University of Missouri-Columbia

Elaine Louder, BS - University of Missouri-Columbia

\section{References}

1. Liston BW, Tartaglia KM, Evans D, Walker C, Torre D. Handoff practices in undergraduate medical education. J Gen Intern Med. 2014;29(5):765-769. doi:10.1007/s11606-014-2806-0

2. Davis R, Davis J, Berg K, et al. Patient Handoff Education: Are Medical Schools Catching Up? Am J Med Qual. 2018;33(2):140-146. doi:10.1177/1062860617719128

3. O'Toole JK, Stevenson AT, Good BP, et al; Initiative for Innovation in Pediatric Education-Pediatric Research in Inpatient Settings Accelerating Safe Signouts Study Group. Closing the gap: a needs assessment of medical students and handoff training. J Pediatr. 2013;162(5):887-8.e1. doi:10.1016/j.jpeds.2013.01.045

4. Starmer AJ, Spector ND, West DC, et al; I-PASS Study Group. Integrating Research, Quality Improvement, and Medical Education for Better Handoffs and Safer Care: Disseminating, Adapting, and Implementing the I-PASS Program. Jt Comm J Qual Patient Saf. 2017;43(7):319-329. doi:10.1016/j.jcjq.2017.04.001

5. Melvin L, Cavalcanti RB. The Oral Case Presentation: A Key Tool for Assessment and Teaching in Competency-Based Medical Education. JAMA. 2016;316(21):2187-2188.

doi:10.1001/jama.2016.16415

6. Association of American Medical Colleges. Core Entrustable Professional Activities for Entering Residency (Updated). https://www.mededportal.org/icollaborative/resource/887. Accessed May 19, 2020

7. Jensen AM, Sanders C, Doty J, Higbee D, Rawlings AL. Characterizing information decay in patient handoffs. J Surg Educ. 2014;71(4):480-485. doi:10.1016/j.jsurg.2013.12.002 